\begin{tabular}{|c|c|}
\hline Title & Third-order dispersion in a passively mode locked continuous wave dye laser \\
\hline Author(s) & Torizuka, Kenji; Y amashita, Mikio \\
\hline Citation & $\begin{array}{l}\text { Journal of the Optical Society of A merica B : Optical Physics, 8(12), 2442-2448 } \\
\text { https://doi.org/10.1364/JOSA B.8.002442 }\end{array}$ \\
\hline Issue Date & 1991-12-01 \\
\hline Doc URL & http:/hdl.handle.net/2115/45323 \\
\hline Rights & C 1991 Optical Society of A merica \\
\hline Type & article \\
\hline File Information & JOSA 8-12_2442-2448.pdf \\
\hline
\end{tabular}

Instructions for use 


\title{
Third-order dispersion in a passively mode-locked continuous-wave dye laser
}

\author{
Kenji Torizuka and Mikio Yamashita \\ Laser Section, Opto-Electronics Division, Electrotechnical Laboratory, 1-1-4 Umezono, Tsukuba, \\ Ibaraki 305, Japan
}

Received December 14, 1990; revised manuscript received July 26, 1991

\begin{abstract}
The pulse duration $t_{p}$ of a colliding-pulse mode-locked dye laser is measured versus the intracavity third-order dispersion (TOD), where the group-velocity dispersion is optimized by a four-prism sequence. The intracavity TOD is varied by the addition of layers on quarter-wave dielectric-multilayer mirrors. The shortest pulse is observed at a nonzero TOD. The TOD dependence of $t_{p}$ is explained by a new model that describes the saturation of absorption and gain dyes in the frequency domain, with the assumption of a steady $\operatorname{sech}^{2}$-shaped pulse with weak chirp and a negligible fast self-phase modulation.
\end{abstract}

\section{INTRODUCTION}

Recent advances in the development of femtosecond pulse lasers showed that tuning the intracavity group-velocity dispersion (GVD) effectively shortens the pulse duration $t_{p}$. The tuning is done by means of two kinds of dispersive device, refractive (a glass plate or Brewster prisms ${ }^{1,2}$ ) and interferometric [a Gires-Tournois (GT) interferometer ${ }^{3}$ or periodic dielectric-multilayer coatings ${ }^{4-6}$ on cavity mirrors]. The circulating pulse in a passively mode-locked cw dye laser is formed and modified by the saturable gain and absorption, the intracavity dispersion, and an intensity-dependent refractive index of the dye solvent. The gain and absorption are the sources of the slow selfphase modulation (SPM), and the refractive index is the source of the fast SPM. The GVD-tuning devices compensate for not only GVD that is due to the intracavity elements but also a linear chirp that is due to the dominant fast SPM, as was shown by Valdmanis and co-workers. ${ }^{2,7,8}$

Analysis of pulse generation in a passively mode-locked dye laser is generally quite complicated. Many theoretical studies were carried out for stationary mode-locking by pulse amplitude modulation, ${ }^{9-12}$ an effect of the intracavity GVD and the fast SPM. ${ }^{13-15}$ Furthermore, it was shown that a critical analysis of nonstationary mode locking and solitonlike pulse formation ${ }^{16-19}$ is necessary when the fast SPM is significant. When the fast SPM is weak, both saturable dyes form a stationary pulse train. ${ }^{20,21}$

Several authors pointed out that pulse shortening is limited by the frequency-dependent GVD (the higherorder dispersion). ${ }^{5,22,23}$ The lowest-order term of the frequency-dependent GVD is called the third-order dispersion (TOD). Khühlke et $a l^{22}$ first observed the effect of the frequency-dependent GVD on $t_{p}$ and the pulse fluctuation in the colliding-pulse mode-locked (CPM) laser that contains both the four-prism sequence and the GT interferometers. However, the frequency-dependent GVD of their GT interferometers was so strong that it was not accounted for by TOD alone. Therefore the exact TOD dependence of $t_{p}$ was not obtained.

Recently the numerical simulation by de Barros et al..$^{24}$ showed that the optimization of TOD shortens the pulses of a GVD-adjusted CPM laser. The more recent experimental work by Goto et $\mathrm{al}^{25}$ resulted in the generation of a 22-25-fs pulse with a combination of the cavity mirrors with TOD and the four-prism sequence. Salin et al. ${ }^{26}$ studied the TOD effect in solitonlike pulse formation. In spite of these investigations, a clear quantitative relation between the intracavity TOD and passive mode-locking parameters has not yet been established experimentally and theoretically.

In this paper the TOD dependence of $t_{p}$ is measured with good reproducibility in a GVD-adjusted CPM dye laser during weakly saturated absorption by using a combination of the cavity mirrors with additional layers for TOD tuning and the four-prism sequences. It is found that the pulse duration is broadened by a large positive and negative TOD and is minimized at nonzero TOD. The measured TOD dependence is explained by a new analysis with a frequency-domain model for a weakly chirped pulse with a sech ${ }^{2}$ shape. The stationary mode locking under the weak fast SPM is treated by the model. It is shown that the TOD that generates the shortest pulse becomes of nonzero value because of the frequencydependent phase delay caused by dye saturations and a finite band-limiting loss.

\section{EXPERIMENTS}

\section{A. Tuning of Intracavity Third-Order Dispersion}

First we describe the tuning of the intracavity dispersion of the CPM laser used for the experiment, where specially designed cavity mirrors and a sequence of four Brewster prisms (B.P.'s) are employed as dispersion-tuning elements (Fig. 1). The phase delay caused by these dispersive elements for one turn of the ring cavity is denoted as $\varphi(\omega)$. It is expanded to a Taylor series as $\varphi(\omega)=\varphi^{(0)}+$ $\varphi^{(1)} \Delta \omega+\left[\varphi^{(2)} / 2\right] \Delta \omega^{2}+\left[\varphi^{(3)} / 6\right] \Delta \omega^{3}+\delta \varphi(\omega)$, where $\varphi^{(n)}$ is the $n$ th-order derivative of the phase delay with respect to the angular frequency $\omega(n=0,1,2,3), \delta \varphi(\omega)$ is the higherorder term that is due to the mirrors and prism sequence, and $\Delta \omega=\omega-\omega_{0}$ with lasing center angular frequency $\omega_{0}$. Accordingly, $\varphi^{(n)}$ and $\delta \varphi(\omega)$ are $\varphi^{(n)}=\Sigma \varphi_{\mathrm{ps}}^{(n)}+\Sigma \varphi_{i}^{(n)}$ 


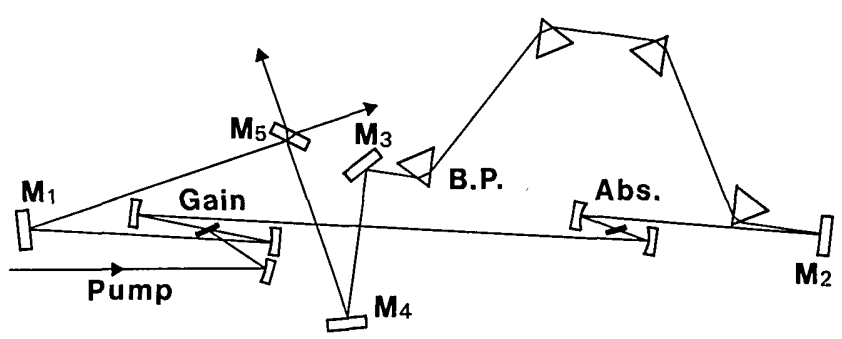

Fig. 1. Schematic diagram of the cavity configurations. The ring cavity-length is $\sim 4 \mathrm{~m}$ (Abs., absorber).

and $\delta \varphi=\delta \varphi_{\mathrm{ps}}+\Sigma_{i} \delta \varphi_{\mathrm{i}}$, where the subscripts ps and $i$ specify the contributions of the prism sequence and each of the cavity mirrors. The dispersive contributions of the transient gain and absorption are excluded from $\varphi(\omega)$. Following the notation of Fork et al., ${ }^{10} \mathrm{GVD}$ (the quadratic phase) and TOD (the cubic phase) are proportional to $-\varphi^{(2)}$ and $-\varphi^{(3)}$, respectively.

The second-order dispersion and TOD's of the fourprism sequence $\left(\varphi_{\mathrm{ps}}^{(2)}\right.$ and $\left.\varphi_{\mathrm{ps}}^{(3)}\right)$ are calculated to be $3.1 \times l p-52 \times l m\left(\mathrm{fs}^{2}\right)$ and $4.7 \times l p-24 \times l m\left(\mathrm{fs}^{3}\right)$ at $632 \mathrm{~nm}$, respectively. ${ }^{10}$ Here $l_{p}(\mathrm{~mm})$ and $l_{m}(\mathrm{~mm})$ are the prism separation and the total material length through the fused-quartz prisms. Because of the finite beam radius and spectral width of the laser, $l_{m}$ must be greater than a few millimeters. Therefore $\varphi_{\mathrm{ps}}^{(3)}$ is positive $\left(\gtrsim 300 \mathrm{fs}^{3}\right.$ ) when $\varphi_{\mathrm{ps}}^{(2)} \gtrsim 0$. In the experiments $l_{m}$ is estimated by the observation of spacings between two beams reflected from the surfaces of each prism. The variation of $l_{m}$ is measured more precisely by displacements of the translation stages for prism insertion.

Our dispersion-designed mirrors consist of double stacks of additional dispersive layers (upper side) and high-reflection quarter-wave layers for the lasing frequency (lower side). Each mirror is designed for use at either $45^{\circ}$ ( $p$ polarization) or $0^{\circ}$. At $632 \mathrm{~nm}$ mirror number $i$ satisfies $\varphi_{i}^{(2)}>0, \varphi_{i}^{(3)}<0$, and $\delta \varphi_{i} \sim 0$.

In all the mirrors $\mathrm{TiO}_{2}$ is used as a high-refraction material with refractive index $n_{H}$ and $\mathrm{SiO}_{2}$ as a lowrefraction material with refractive index $n_{L}$. The multilayer theory predicts that a double-stacked mirror with an upper stack of periodic layers, $\left[H\left(0.188 \lambda_{0}\right)-\right.$ $\left.L\left(0.188 \lambda_{0}\right)\right]^{3}$, and a lower stack of high-reflection layers, $\left[H\left(\lambda_{0} / 4\right)-L\left(\lambda_{0} / 4\right)\right]^{n}$, will give a dispersion comparable with that of a GT interferometer, $\left[H\left(0.25 \lambda_{0}\right)-L\left(\lambda_{0}\right)\right]-$ $\left.H\left(\lambda_{0} / 4\right)-L\left(\lambda_{0} / 4\right)\right]^{n}$. Here $H\left(n_{H} d_{H}\right)$ and $L\left(n_{L} d_{L}\right)$, respectively, denote the high- and low-refraction layers with the optical thicknesses $n_{H} d_{H}$ and $n_{L} d_{L}$, and $\lambda_{0}$ is the lasing center wavelength at $\sim 632 \mathrm{~nm}$. We use this doublestacked mirror because it can be easily manufactured by mirror suppliers with a guaranteed quality. Careful attention in the manufacturing process is also paid to the accuracy of the layer thickness and the exclusion of impurities in order to minimize dispersion error and additional absorption.

We discuss $\delta \varphi(\omega)$ of mirrors a, $\mathrm{a}^{\prime}$, and b, employed for the TOD tuning, whose layer compositions were described previously. ${ }^{6}$ Mirrors a, $\mathrm{a}^{\prime}$, and b are composed of double stacks of

$\left[L\left(0.206 \lambda_{0}\right)-H\left(0.206 \lambda_{0}\right)\right]^{3}-\left[L\left(0.264 \lambda_{0}\right)-H\left(0.264 \lambda_{0}\right)\right]^{12}$, $\left[L\left(0.177 \lambda_{0}\right)-H\left(0.177 \lambda_{0}\right)\right]^{3}-\left[L\left(0.248 \lambda_{0}\right)-H\left(0.248 \lambda_{0}\right)\right]^{12}$, $\left[L\left(0.193 \lambda_{0}\right)-H\left(0.193 \lambda_{0}\right)\right]^{4}-\left[L\left(0.237 \lambda_{0}\right)-H\left(0.237 \lambda_{0}\right)\right]^{12}$, respectively. In Fig. 2 the frequency dependences of $\varphi_{i}^{(2)}$ and $\varphi_{i}^{(3)}$ for mirrors $\mathrm{a}$ and $\mathrm{b}$ are shown. Mirrors $\mathrm{a}$ and $\mathrm{b}$ give the largest $\left|\varphi_{i}^{(3)}\right|$ of all the mirrors for the incident angles of $45^{\circ}$ and $0^{\circ}$, respectively. The dispersion characteristics of mirror $a^{\prime}$, employed for the nearly normal incidence, correspond to those of mirror a at $45^{\circ}$. Figure 3 shows the frequency dependence of $\delta \varphi_{i}(\omega)$ at a center frequency $\omega_{0}=2.98 \times 10^{15} \mathrm{rad} / \mathrm{s}$ for mirrors $\mathrm{a}$ and $\mathrm{b}$. The same combinations of mirrors $a, a^{\prime}$, and $b$ for $M_{1}-M_{4}$ in Fig. 1 are employed to vary the value of the TOD, keeping $\Sigma_{i} \delta \varphi_{i}(\omega)$ minimal. It will be confirmed that $\delta \varphi(\omega)$ $\left[\sim \Sigma_{\mathrm{i}} \delta \varphi_{\mathrm{i}}(\omega)\right]$ is negligibly small within the bandwidths of the mode-locked laser pulses, as is shown in Subsection 2.B. However, it should be noted that $\delta \varphi(\omega)$ is not sufficiently small for the shortest pulses of a GVDadjusted CPM dye laser. For example, to obtain a 30-fs pulse, one must tune $\varphi^{(2)}$ and $\varphi^{(3)}$ within $1 \mathrm{fs}^{2}$ and $100 \mathrm{fs}^{3}$, respectively. At the same time, $\delta \varphi(\omega)$ should be less than $\sim 0.5 \times 10^{-3}$ rad within the corresponding bandwidth.

\section{B. Measurement of the Third-Order-Dispersion Dependence of the Pulse Duration}

The cavity of the CPM laser that is used for the experiment (Fig. 1) consists of a pair of $f=50 \mathrm{~mm}$ concave mirrors, with a mixture of Rhodamine 6G (Rh6G) and Kiton Red as a gain medium, a pair of $f=25 \mathrm{~mm}$ concave mirrors with diethyloxadicarbocyanine iodide (DODCI) as an absorber medium, a four-prism sequence, and the five

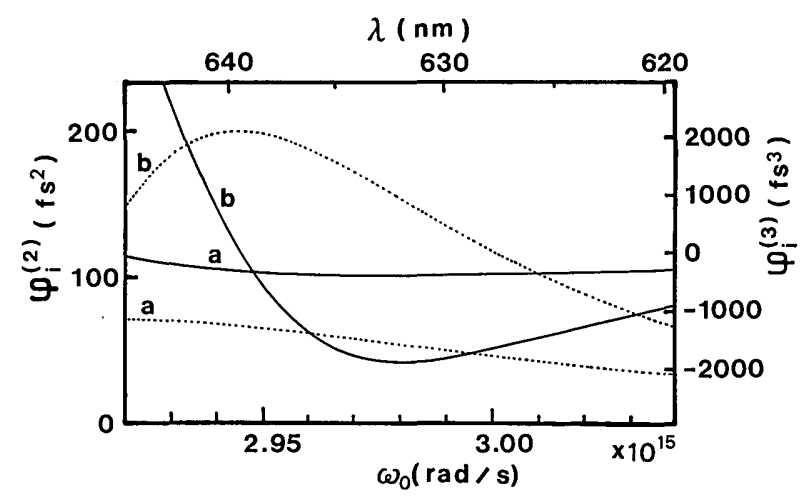

Fig. 2. Calculated frequency dependencies of the second-order dispersion $\varphi_{i}^{(2)}$ (dashed curves) and the TOD's $\varphi_{i}^{(3)}$ (solid curves) of TOD-tuning mirrors $a$ and $b$.

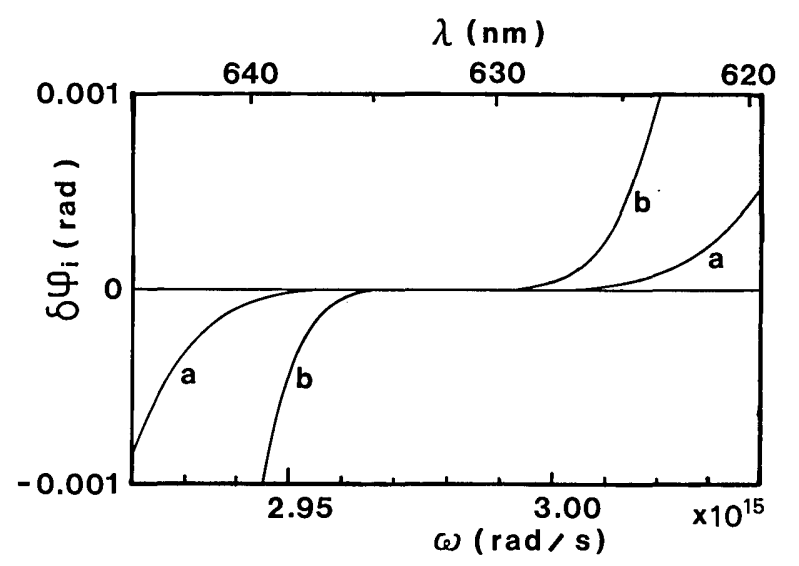

Fig. 3. Higher-order dispersions in the expansions at frequency $\omega_{0}=2.98 \times 10^{15} \mathrm{rad} / \mathrm{s}$. Mirrors $\mathrm{a}$ and $\mathrm{b}$ are the same as those in Fig. 2. 


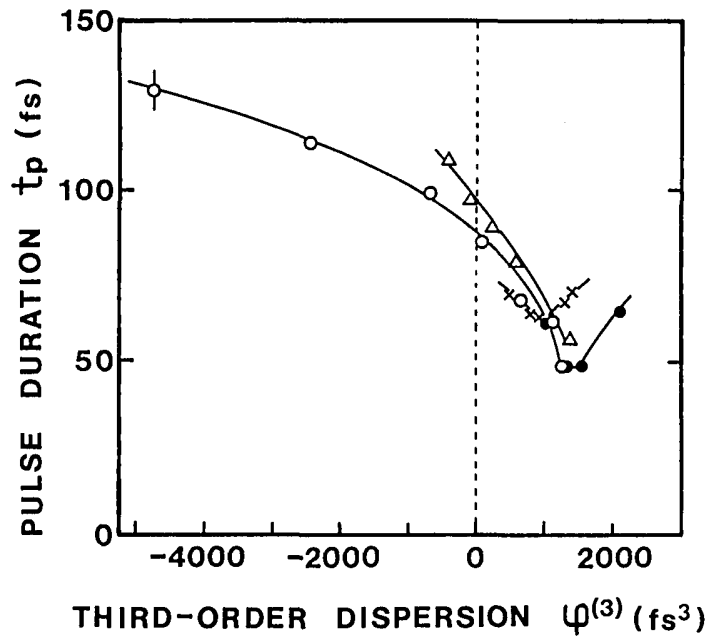

Fig. 4. Pulse duration $t_{p}$ versus the negative TOD $\varphi^{(3)}$. Gain dye, Rh6G $2.9 \mathrm{mM}$ : Kiton Red $0.8 \mathrm{mM}$; saturable absorber, DODCI $1.0 \mathrm{mM}$ (triangles), $1.5 \mathrm{mM}$ (circles), and $3.8 \mathrm{mM}$ (crosses).

plane mirrors $M_{1}-M_{5}$. Mirrors $M_{1}, M_{2}$, and $M_{4}$ are used at nearly normal incidence. Mirrors $\mathrm{M}_{3}$ and $\mathrm{M}_{5}$ (the output coupler) are used at $45^{\circ}$ ( $p$ polarization). The dispersion of the all-concave mirrors is negligibly small. The output coupler contributes a constant small dispersion $\left(\varphi_{\mathrm{oc}}^{(2)}=3.2 \mathrm{fs}^{2}\right.$ and $\left.\varphi_{\mathrm{oc}}^{(3)}=31 \mathrm{fs}^{3}\right)$. An angle-tuning technique $^{3-5}$ is not used in this experiment. The dispersiondesigned mirrors $a, a^{\prime}$, and $b$ are employed for $M_{1}-M_{4}$.

The transmission $T$ of the output coupler is $3 \%$ at $632 \mathrm{~nm}$, and its minimum is at $600 \mathrm{~nm}$. Such a slightly blue-shifted output coupler gives stable mode locking and good reproducibility in the TOD-dependence measurement. The addition of Kiton Red to the Rh6G solution decreases the laser threshold and the circulating pulse energy. The average output is $7 \mathrm{~mW}$, with $1.5 \mathrm{~W}(514.5 \mathrm{~nm})$ for pumping at the 1.5-mM DODCI concentration. The jet-sheet thicknesses are $150 \mu \mathrm{m}$ for the gain medium and $40 \mu \mathrm{m}$ for the absorber. For this operating condition the features that arise from the strong fast SPM (a sensitive jet position and a spiky structure of the laser spectrum ${ }^{2}$ ) are not seen when the TOD dependence of the pulse duration is measured. On the other hand, a further pulse shortening that accompanies these features is observed with the small TOD, a normal-incidence output coupler $\mathrm{M}_{2}$ $(T=1 \%)$, no Kiton Red, and a higher pumping power.

The total material length $l_{m}$ of the prisms is adjusted for the GVD compensation and the generation of the shortest pulse while we monitor the spectrum and autocorrelation trace. The shortest pulse is observed slightly before the abrupt change of the spectral and correlation shapes occurs. Figure 4 shows $t_{p}$ versus $\varphi^{(3)}$, where $l_{m}$ is adjusted to generate the shortest pulse and optimize the intracavity GVD at each point of the various TOD's. Each data point indicates the average of several sets of slow-scan autocorrelation measurements. The value of $t_{p}$ is the FWHM of the intensity on the assumption of a nonchirped $\operatorname{sech}^{2}$ shaped pulse. The open circles in Fig. 4 represent the TOD dependence measured at the 1.5-mM DODCI concentration by the combination of dispersive mirrors $a, a^{\prime}, b$ with prism separation $l_{p}=380 \mathrm{~mm}$. The triangles (1.0-mM DODCI concentration) and the crosses (3.80-mM DODCI concentration) represent the data measured at $l_{p}=450 \mathrm{~mm}$. For the filled circles (1.5-mM DODCI) $l_{p}$ and $l_{m}$ are varied, and $\mathrm{M}_{1}-\mathrm{M}_{4}$ are the quarter-wave mirrors with negligible dispersions. For all the data points the center wavelength is $632 \pm 2 \mathrm{~nm}$ with a bell-shaped pulse spectrum, and the width is near the transform limit of a nonchirped $\operatorname{sech}^{2}$ pulse (the measured widest spectral width is $9.1 \mathrm{~nm}$ ).

The result shows that, in the negative- $\varphi^{(3)}$ region, pulse duration $t_{p}$ increases as $\varphi^{(3)}$ decreases (the open circles in Fig. 4). The curves (the crosses and the filled circles in Fig. 4) indicate that the minimum $t_{p}$ exists at the positive $\varphi^{(3)}$ (negative TOD). The optimum value, $\varphi_{0}^{(3)}$, is $+1000 \sim 1500 \mathrm{fs}^{3}$. When $\varphi^{(3)}$ is larger than $\varphi_{0}^{(3)}$, however, the tails of the autocorrelation traces of the generated pulses gradually increase as $\varphi^{(3)}$ increases. The reliability of the data is confirmed by the fact that all the TOD dependences of $t_{p}$ are repeatedly obtained within an accuracy of $\pm 5 \%$ (the error bar) when all the dyes are fresh. Furthermore, the result is reproduced when the whole laser cavity is taken to pieces and reconstructed.

There are no measurable differences among the laser thresholds for the different combinations of the dispersive mirrors after the optimization of $\varphi^{(2)}$ by the prism insertion. Therefore the additional loss that is due to the dispersive layers is considered to be sufficiently small. The optimal intracavity GVD is nearly constant at any DODCI concentration in the measured $\varphi^{(3)}$ range. The adjustment of $l_{m}$ for the shortest pulse compensates for the difference of $\varphi_{i}^{(2)}$ between the exchanged mirrors. This fact also confirms that the practical dispersions of the used mirrors coincide with the designed values.

\section{THEORETICAL CONSIDERATIONS}

Generally the relation between the pulse duration $\left(t_{p}\right)$ and the intracavity TOD $\left(-\varphi^{(3)}\right)$ is treated by a numerical simulation, ${ }^{24}$ since a passively mode-locked laser involves a balance of many factors. ${ }^{12-16,20}$ However, the situation for our experiment makes it possible to analyze the experimental result by a following simplified model in the frequency domain. That is, under the present TODdependence measurement, pulse shaping by the fast SPM is weak. Thus the TOD-dependence of the pulse duration should be explained by the contributions of both saturable dyes.

\section{A. Fundamental Equations in the Frequency Domain}

In the group-velocity coordinate the electric field $\mathscr{E}(t)$ of the $\operatorname{sech}^{2}$ pulse in the slowly varying envelope approximation is represented as

$$
\mathscr{E}(t)=E(t) \exp \left(i \omega_{0} t\right)+E^{*}(t) \exp \left(-i \omega_{0} t\right)
$$

with

$$
E(t)=E_{0}[\operatorname{sech}(t / \tau)] \exp [i \Phi(t / \tau)],
$$

where $\Phi(t / \tau)$ is the slowly varying phase and $\tau=t_{p} / 1.76$. In the following discussion a weak-chirp model is adopted:

$$
\exp [i \Phi(t / \tau)]=1+i\left[\frac{c_{2}}{2}\left(\frac{t}{\tau}\right)^{2}+\frac{c_{3}}{6}\left(\frac{t}{\tau}\right)^{3}\right]
$$

Here $c_{2}$ and $c_{3}$ denote the linear- and parabolic-chirp coefficients, respectively, satisfying $\left|c_{2} / 2\right|,\left|c_{3} / 6\right| \ll 1$. 
Gain Medium

\section{Absorber}

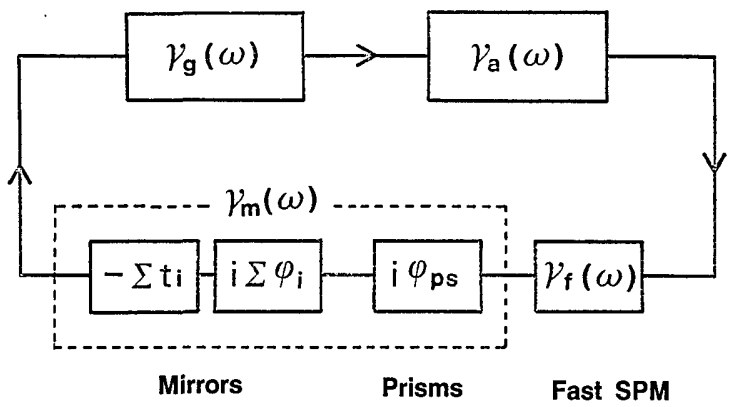

Fig. 5. Schematic diagram of the analytical model. The complex propagators of the saturable gain $\gamma_{g}$, the saturable absorption $\gamma_{a}$, and the nonlinear refractive material $\gamma_{f}$ are dependent on the pulse amplitude and phase. The cavity loss is mainly due to the transmission $\Sigma_{i} t_{i}$ of the mirrors. The phase delay of the mirrors $\Sigma_{i} \varphi_{i}$ and the prism sequence $\varphi_{\mathrm{ps}}$ are controlled in the experiments.

A Fourier component $\tilde{E}(\omega)$ of the field amplitude is represented as a function of the detuning $\Delta \omega\left(\equiv \omega-\omega_{0}\right)$, $\tilde{E}(\omega) \equiv \int_{-\infty}^{\infty} E(t) \exp (-i \Delta \omega t) \mathrm{d} t$. When the pulse passes through each optical element, $\tilde{E}(\omega)$ is modified to $\tilde{E}^{\prime}(\omega)=\tilde{E}(\omega) \exp \left[\gamma_{j}(\omega)\right]$, where $\gamma_{j}(\omega)(j=g, a, f, m)$ are the complex propagators in the frequency domain (Fig. 5). Here $\gamma_{g}(\omega), \gamma_{a}(\omega), \gamma_{f}(\omega)$, and $\gamma_{m}(\omega)$ represent, respectively, the effect of the saturable gain, the saturable absorption, the fast SPM, and the contribution from the other optical components, including the dispersion-tuning elements.

Assuming that the pulse is only slightly altered with regard to any one element on a single pass, we treat only the lowest-order term for the propagators. This means that strong modulation phenomena, such as solitonlike pulse shaping, ${ }^{16}$ are not treated. The assumption also allows us to derive the $\gamma_{j}$ of each element independently $\left[\gamma_{j}(\omega)\right.$ is referred to hereafter as $\gamma_{j}$ ].

For homogeneously broadened dyes ${ }^{17} \gamma_{g}$ and $\gamma_{a}$ are represented as

$$
\begin{aligned}
& \gamma_{g}=\frac{\alpha_{g}+i d_{g} \alpha_{g}}{\tilde{E}(\omega)} \int_{-\infty}^{\infty} \mathrm{d} t E(t) \exp \left[-\frac{\Gamma(t)}{\Gamma_{g}}-i \Delta \omega t\right], \\
& \gamma_{a}=\frac{-\alpha_{a}-i d_{a} \alpha_{a}}{\tilde{E}(\omega)} \int_{-\infty}^{\infty} \mathrm{d} t E(t) \exp \left[-\frac{\Gamma(t)}{\Gamma_{a}}-i \Delta \omega t\right],
\end{aligned}
$$

where

$$
\Gamma(t)=\int_{-\infty}^{t} \mathrm{~d} t^{\prime}\left(t^{\prime}\right)
$$

is the cumulative energy in the pulse with the intensity $I(t)\left(\propto|\mathscr{E}(t)|^{2}\right)$ and the total energy $\Gamma_{T}=\Gamma(\infty)$. The detunings $d_{g}\left(\right.$ or $\left.d_{a}\right)$ are defined as $d_{g(a)}=2\left(\omega_{g(a)}-\omega_{0}\right) / \Delta \omega_{g(a)}$, where $\omega_{g(a)}$ and $\Delta \omega_{g(a)}$ are the center wavelength and FWHM of the gain (or the absorption) band. On the assumption of sufficiently broad gain and absorption bands $\left(\Delta \omega_{g}\right.$ and $\left.\Delta \omega_{a} \gg 1 / \tau\right), \alpha_{g}$ and $\alpha_{a}$ are the amplitude gain and the amplitude absorption at the frequency $\omega_{0}$ before arrival of the pulse, respectively. In addition, $\Gamma_{g}$ and $\Gamma_{a}$ are the saturation energies of the gain and absorption media.

The phase retardation by the weak fast SPM ${ }^{10}$ is $\varphi_{f}(t)=-n_{2} I(t) \omega_{0} \Delta l / c$ with the nonlinear refractive index $n_{2}$ and the medium thickness $\Delta l$. Therefore $\gamma_{f}$ is repre- sented as

$$
\gamma_{f}=-i \frac{\kappa}{\tilde{E}(\omega)} \int_{-\infty}^{\infty} \mathrm{d} t E(t) I(t) \exp (-i \Delta \omega t)
$$

where $\kappa=n_{2} \omega_{0} \Delta l / c$.

We describe $\gamma_{m}\left(=\gamma^{\prime}+i \gamma^{\prime \prime}\right)$ in terms of the amplitude transmission coefficients $\Sigma_{i} t_{i}$ of the cavity mirrors $(i)$ and the sum of their dispersions $\Sigma_{i} \varphi_{i}$ and the prism sequence dispersion $\varphi_{\mathrm{ps}}$ as

$$
\gamma^{\prime}=-\sum_{i} t_{i}, \quad \gamma^{\prime \prime}=\varphi_{\mathrm{ps}}+\sum_{i} \varphi_{i}
$$

The $n$ th-order Taylor coefficients on $\Delta \omega$ of $\gamma^{\prime}, \gamma^{\prime \prime}, t_{i}, \varphi_{i}$, and $\varphi_{\mathrm{ps}}$ are denoted by $\gamma^{\prime(n)}, \gamma^{\prime(n)}, t_{i}^{(n)}, \varphi_{i}^{(n)}$, and $\varphi_{\mathrm{ps}}^{(n)}$, respectively. Then $\gamma^{\prime \prime(2)}$ and $\gamma^{\prime \prime(3)}$, respectively, are equal to $\varphi^{(2)}$ and $\varphi^{(3)}$ in our experiment.

In stationary mode locking no change occurs in the pulse except that there is a constant phase $a$ and group delay $b \tau$. The steady-state consistency requirements lead to

$$
\gamma_{g}+\gamma_{a}+\gamma_{f}+\gamma_{m}=i(a+b \tau \Delta \omega),
$$

where $a$ and $b$ are any real values. By a Taylor expansion of Eq. (5) to the third order on $\Delta \omega$, we obtain six simultaneous equations, which represent the balances of the amplitude terms of zeroth, first, second, and third orders [corresponding to Eqs. (6)-(8) and (10)] and the phase terms of the second and the third orders [Eqs. (9) and (11)].

For weak-chirp pulses, represented by Eq. (2), with the weak fast SPM we can further simplify the six simultaneous equations to the following forms:

$$
\begin{aligned}
P_{1}+\left(c_{2} / 2\right) P_{2}+\left(c_{3} / 6\right) P_{3}+\gamma^{\prime(0)} & =0, \\
Q_{1}+\left(c_{2} / 2\right) Q_{2}+\left(c_{3} / 6\right) Q_{3}+\left[\gamma^{\prime(1)} / \tau\right] & =0, \\
R_{1}+\left(c_{2} / 2\right) R_{2}+\left(c_{3} / 6\right) R_{3}+\left[\gamma^{\prime(2)} / \tau^{2}\right] & =0, \\
S_{1}+\left(c_{2} / 2\right) S_{2}+\left(c_{3} / 6\right)-\left(\kappa \Gamma_{T} / 2 \tau\right)+\left[\varphi^{(2)} / \tau^{2}\right] & =0, \\
T_{1}+\left(c_{2} / 2\right) T_{2}+\left(c_{3} / 6\right) T_{3}+\left[\gamma^{\prime(3)} / \tau^{3}\right] & =0, \\
U_{1}+\left(c_{2} / 2\right) U_{2}+\left(c_{3} / 6\right) U_{3}+\left[\varphi^{(3)} / \tau^{3}\right] & =0,
\end{aligned}
$$

where $P_{i}, Q_{i}, R_{i}, S_{i}, T_{i}$, and $U_{i}(i=1,2,3)$ are given in Appendix A.

Here we assume that the lasing center frequency $\omega_{0}$ and the laser power are nearly independent of $\varphi^{(3)}$ and that the chirping is weak [after the optimization of $\varphi^{(2)}$. For the present laser operation the assumptions are confirmed to be reasonable by experiment. This enables us to solve Eqs. (8)-(11) with the empirical values of a constant pulse energy $\Gamma_{T}$ and laser frequency $\omega_{0}$ instead of those values calculated from Eqs. (6) and (7).

\section{B. Group-Velocity-Dispersion Dependence of Pulse}

Duration

The dependences of $\tau$ and $c_{2}$ on the intracavity GVD $\left[-\varphi^{(2)}\right]$ are derived by using Eqs. (8) and (9) for the second-order terms with neglect of $c_{3}$. For example, $\tau$ is represented as

$$
\tau^{2}-\left[-\kappa \Gamma_{T} \tau+\varphi^{(2)}\right] p+q \gamma^{\prime(2)}=0,
$$

where

$$
p=\frac{R_{2}}{R_{1} S_{2}-R_{2} S_{1}}, \quad q=\frac{S_{2}}{R_{1} S_{2}-R_{2} S_{1}} .
$$




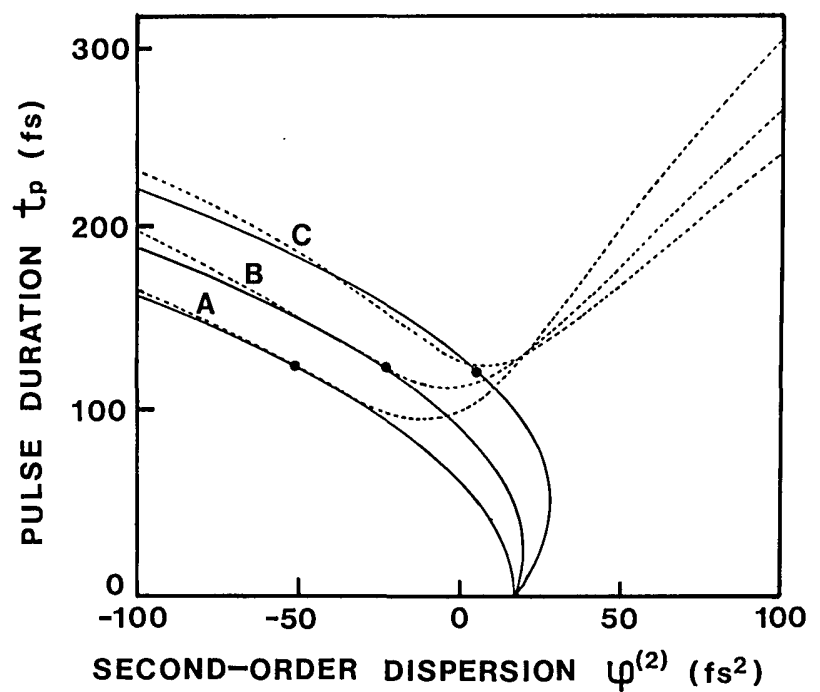

Fig. 6. Pulse durations versus GVD, calculated by the frequencydomain model for the present CPM dye laser. In addition to the results obtained for the weak-chirp model of Eq. (2) (solid curves), the results for the usual chirping model (see text; dashed curves) with various fast SPM's and $\kappa \Gamma_{T}$ equal to $0,0.4$, and $0.8 \mathrm{fs}$ are shown as curves $A, B$, and $C$, respectively.

The solid curves in Fig. 6 show the $\varphi^{(2)}$ dependence of $\tau$ [Eq. (12)] for the present CPM dye laser, where we estimate $\omega_{0}=2.98 \times 10^{15} \mathrm{rad} / \mathrm{s}, \Gamma_{T} / \Gamma_{a}=6.4, \Gamma_{T} / \Gamma_{g}=0.8$, $\alpha_{g}=0.041, \alpha_{a}=0.016, \gamma^{\prime(0)}=-0.025$, and $\gamma^{\prime(2)}=-30.0 \mathrm{fs}^{2}$. The CPM effect is taken into account ${ }^{15}$ in the estimation of $\Gamma_{a}$. We obtain $p=-72$ and $q=41$ from Eq. (13), since $\omega_{a}=3.20 \times 10^{15} \mathrm{rad} / \mathrm{s}$ and $\omega_{g}=$ $3.32 \times 10^{15} \mathrm{rad} / \mathrm{s}$ with, from Ref. $13, \Delta \omega_{a}=0.22 \times$ $10^{15} \mathrm{rad} / \mathrm{s}$ and $\Delta \omega_{g}=0.24 \times 10^{15} \mathrm{rad} / \mathrm{s}$ for $R h 6 \mathrm{G}$ and DODCI. $^{13}$ For curves A, B, and C the fast-SPM coefficients $\kappa \Gamma_{T}$ are equal to $0,0.4$, and $0.8 \mathrm{fs}$, respectively. The filled circles on the curves indicate the nonchirped pulses $\left(c_{2}=0\right)$.

To estimate the range of $c_{2}$ for which the model of Eq. (12) is reasonable, the numerical results of the frequency-domain approach for a typical non-weak-chirp pulse, ${ }^{14} \exp [i \Phi(t / \tau)]=\left[\operatorname{sech}(t / \tau]^{-i c_{2}}\right.$ in Eq. (1), are also shown in Fig. 6 as the dashed curves. The curves are obtained by a complete calculation of $\Delta \omega^{2}$ terms in Eqs. (3) and (4) with assumptions of constant $\Gamma_{T}$ and $\omega_{0}$. When $\kappa \Gamma_{T}=0$, the solid curves of Eq. (12) almost agree with the dashed curves when $\left|c_{2} / 2\right| \leq 0.4$. The minimum pulse duration is found on the dashed curve, which is consistent with the usual chirp-compensation model. ${ }^{1}$ It should be noted that the shape of the dashed curve for positive $\varphi^{(2)}$ (with large $c_{2}$ ) will be influenced more sensitively than that for negative $\varphi^{(2)}$ by the neglected chirp-dependent frequency shift.

The direction of the parabola in the $\varphi^{(2)}, \tau$ plane is determined by the sign of $p$. When $\omega_{0}$ is on the blue side of $\omega_{a}$, $p$ is positive. Then weakly chirped pulses should exist under positive $\varphi^{(2)}$ (negative GVD). This fact is in agreement with the analysis in the time domain of Ref. 18. In addition, from Eqs. (8) and (9), $\tau=\left(-\gamma^{\prime(2)} / R_{1}\right)^{1 / 2}$ when $c_{2}=0$. This means that the duration of the pulse without chirp is independent of the weak fast-SPM $\kappa$, which was first pointed out by Haus and Silberberg. ${ }^{15}$
C. Third-Order-Dispersion Dependence of Pulse Duration To evaluate the TOD dependence, Eqs. (8)-(11) must be solved simultaneously. The parabolic chirp $c_{3}$ and the optimum second-order dispersion $\varphi^{(2)}$ are determined in addition to the linear chirp $c_{2}$ and the pulse duration $\tau$. The derived $\varphi^{(2)}$ corresponds to the intracavity GVD that is adjusted to produce the shortest $\operatorname{sech}^{2}$ pulse at a given TOD in the experiment.

We treat a case of the negligible fast SPM $(\kappa=0)$. Furthermore $\gamma^{\prime(3)} / \tau^{3} \sim 0$ is assumed because the relation $\left|T_{1}\right| \gg\left|\gamma^{\prime(3)} / \tau^{3}\right|$ is satisfied in Eq. (10) for the present laser. With these approximations, the $\varphi^{(3)}$ dependence of $\tau$ is written in a simple equation as

$$
\tau^{3}+r{\tau^{\prime}}^{(2)} \tau+s \varphi^{(3)}=0
$$

where

$$
\begin{aligned}
& r=\left|\begin{array}{ll}
T_{2} & T_{3} \\
U_{2} & U_{3}
\end{array}\right| /\left|\begin{array}{lll}
R_{1} & R_{2} & R_{3} \\
T_{1} & T_{2} & T_{3} \\
U_{1} & U_{2} & U_{3}
\end{array}\right|, \\
& s=\left|\begin{array}{ll}
R_{2} & R_{3} \\
T_{2} & T_{3}
\end{array}\right| /\left|\begin{array}{lll}
R_{1} & R_{2} & R_{3} \\
T_{1} & T_{2} & T_{3} \\
U_{1} & U_{2} & U_{3}
\end{array}\right| .
\end{aligned}
$$

When $\gamma^{\prime(2)}\left(\sim-t_{\mathrm{oc}}^{(2)}\right)<0, r>0$, and $s>0$, Eq. (14) generates the solid curve in Fig. 7. The curve reaches to $\tau_{m}=\tau_{0} / \sqrt{3}$ at the maximum of $\varphi_{0}^{(3)}=2 \tau_{0}^{3} / 3 \sqrt{3} s$, where $\tau_{0}$ $\left[=\left(-r \gamma^{\prime(2)}\right)^{1 / 2}\right]$ is the pulse duration at zero $\operatorname{TOD}\left[\varphi^{(3)}=0\right]$. The curve is meaningful when the weak-chirp approximation is valid. Since the parameters $r$ and $s$ strongly depend on the saturation of the absorber $\left[\Gamma(t) / \Gamma_{a}\right]$ and the gain medium $\left[\Gamma(t) / \Gamma_{g}\right]$, the value of the optimum TOD $\left[\varphi_{0}^{(3)}\right]$ changes remarkably because of both saturation depths.

For the present CPM dye laser (for the circles in Fig. 4) we find that $r=77.1$ and $s=37.5$, from Eq. (15). Therefore Eq. (14) predicts that the pulse duration at the TOD of $\varphi_{0}^{(3)}=1140 \mathrm{fs}^{3}$ will become $t_{p}=1.76 \tau_{m}=49 \mathrm{fs}$. The chirp coefficients at the point $\varphi_{0}^{(3)}$ are found by Eqs. (8),(11), and (14) to be $c_{2} / 2=-0.37$ and $c_{3} / 6=0.08$. The chirp is weak in the range $\tau \gtrsim \tau_{m}$. These results are in good agreement not only with the corresponding experimental value of $50 \mathrm{fs}$ at $\varphi_{0}^{(3)}=1200 \mathrm{fs}^{3}$ but also with the behavior of the TOD dependence given by the open circles

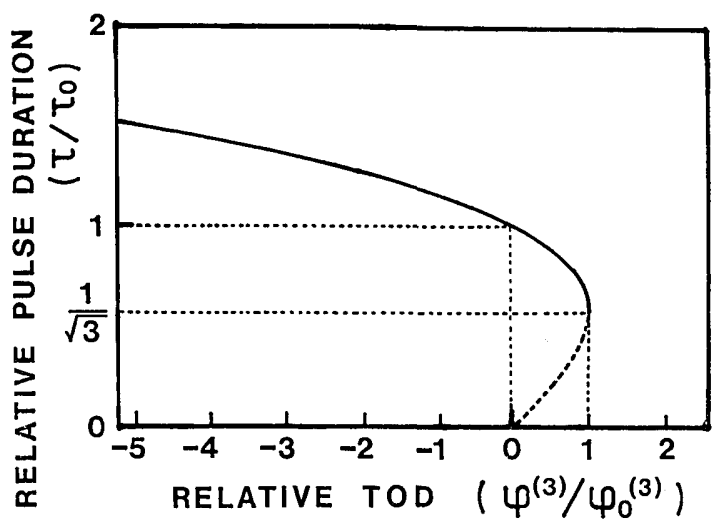

Fig. 7. Theoretical curves for pulse durations versus TOD for positive $r$ and $s$. The normalization factors $\tau_{0}$ and $\varphi_{0}^{(3)}$ are given in the text. 
in Fig. 4 in the TOD region smaller than $\varphi_{0}^{(3)}$. This means that, in the larger region with no $\tau$ solution in Eq. (14), a weakly chirped $\operatorname{sech}^{2}$-shaped pulse does not exist, but a nonsech ${ }^{2}$ or strongly chirped pulse does. This pulse corresponds to the increasing tails of the autocorrelation traces in the positive region over $\varphi_{0}^{(3)}$ that were observed during the measurements represented by crosses and filled circles in Fig. 4.

The existence of the nonzero optimum $\varphi_{0}^{(3)}$ is understood physically as follows. The gain and absorption modulation is forced to balance frequency dependently with the output coupling at the steady state, $-t(\omega)+\operatorname{Re}\left[\gamma_{g}(\omega)\right]$ $+\operatorname{Re}\left[\gamma_{a}(\omega)\right]=0$. Consequently the corresponding phase delay $\operatorname{Im}\left[\gamma_{g}(\omega)\right]+\operatorname{Im}\left[\gamma_{a}(\omega)\right]$ (we call this the dynamic dispersion) also becomes frequency dependent. To generate the shortest pulse, the frequency-dependent phase delay has to be compensated for by the externally adjustable dispersion, $\operatorname{Im}\left[\gamma_{g}(\omega)\right]+\operatorname{Im}\left[\gamma_{a}(\omega)\right]=\Sigma_{i} \varphi_{i}(\omega)+\varphi_{\mathrm{ps}}(\omega)$. Therefore, at the minimum pulse duration $\tau_{m}$, the same amount of TOD is needed $\left[\varphi_{0}^{(3)}=\Sigma_{i} \varphi_{i}^{(3)}+\varphi_{p s}^{(3)} \neq 0\right]$.

\section{CONCLUSIONS}

We have experimentally obtained the pulse duration as a precise function of the TOD $\left[\varphi^{(3)}\right]$ in a CPM dye (Rh6G + DODCI) laser at $\sim 632 \mathrm{~nm}$ for the first time to our knowledge. It has been found that the optimum TOD $\left[\varphi_{0}^{(3)}\right]$ at the point where the shortest pulse duration $\left(\tau_{m}\right)$ is generated is not zero but is positive. This result corresponds to the fact that chirp compensation by the prism sequence usually produces shorter pulses than that by the interferometer, since the former and latter have a positive and a negative $\varphi^{(3)}$, respectively.

It has been shown that an analysis with a frequencydomain description of the slow saturable dyes (in addition to the usual dispersive elements) quantitatively explains the result of the optimum positive $\varphi_{0}^{(3)}$. The reason that the optimum $\varphi_{0}^{(3)}$ becomes positive is that the frequencydependent phase delay caused by the absorption saturation (the dynamic dispersion) yields the dominant negative $\varphi_{0}^{(3)}$ for the shortest pulses generated near $632 \mathrm{~nm}$. It should be noted that one merit of our model, the use of simple parameters, is based on the frequency-domain description of the dynamic dispersion.

As for the behavior of the TOD dependence of the pulse duration in the region from the negative $\varphi_{0}^{(3)}$ to the positive $\varphi_{0}^{(3)}$, our analytical result quantitatively agrees with the experimental one. However, our analysis does not agree with the experimental result for pulse broadening with further increase of the positive $\varphi_{0}^{(3)}$ after the minimal pulse duration $\left(\tau_{m}\right)$ at $\varphi_{0}^{(3)}$. This disagreement is due to our assumption of a weakly chirped sech ${ }^{2}$-pulse. The reason is that when a positive $\varphi_{0}^{(3)}$ is greater than $\varphi_{0}^{(3)}$, the tails of autocorrelation traces in the experiment appear and the pulse shape is slightly modified.

\section{APPENDIX A}

The forms of the coefficients $P_{i}, Q_{i}, R_{i}, S_{i}, T_{i}$, and $U_{i}$ $(i=1,2,3)$ in Eqs. (6)-(11) are given here. They are written as

$$
\begin{aligned}
& P_{i}=P_{i g}-P_{i a}, \quad Q_{i}=Q_{i g}-Q_{i a}, \quad R_{i}=R_{i g}-R_{i a}, \\
& S_{i}=S_{i g}-S_{i a}, \quad T_{i}=T_{i g}-T_{i a}, \quad U_{i}=U_{i g}-U_{i a},
\end{aligned}
$$

where the subscripts $g$ and $a$ denote the gain dye and the absorber, respectively. Introducing $R_{g}^{(n)}$ and $R_{a}^{(n)}$ as

$$
R_{g, a}^{(n)} \equiv \frac{1}{\pi} \int_{-\infty}^{\infty} \mathrm{d} \eta \eta^{n}(\operatorname{sech} \eta) \exp \left[-\frac{\Gamma_{T}}{2 \Gamma_{g, a}}(1+\tanh \eta)\right]
$$

with the normalized time $\eta$ ( $\equiv t / \tau)$, we write the terms for a gain dye in the following equations:

$$
\begin{aligned}
P_{1 g}= & \alpha_{g} R_{g}^{(0)}, \quad P_{2 g}=-\alpha_{g} d_{g}\left[R_{g}^{(2)}-(\pi / 2)^{2} R_{g}^{(0)}\right], \\
P_{3 g}= & -\alpha_{g} d_{g} R_{g}^{(3)}, \quad Q_{1 g}=\alpha_{g} d_{g} R_{g}^{(1)}, \\
Q_{2 g}= & \alpha_{g}\left[R_{g}^{(3)}-(\pi / 2)^{2} R_{g}^{(1)}\right], \quad Q_{3 g}=\alpha_{g}\left[R_{g}^{(4)}-5(\pi / 2)^{4} R_{g}^{(0)}\right], \\
R_{1 g}= & -\alpha_{g}\left[R_{g}^{(2)}-(\pi / 2)^{2} R_{g}^{(0)}\right], \\
S_{2 g}= & -\alpha_{g}\left[R_{g}^{(4)}-2(\pi / 2)^{2} R_{g}^{(2)}-3(\pi / 2)^{4} R_{g}^{(0)}\right], \\
S_{3 g}= & -\alpha_{g}\left[R_{g}^{(5)}-(\pi / 2)^{2} R_{g}^{(3)}-10(\pi / 2)^{4} R_{g}^{(1)}\right], \\
S_{1 g}= & d_{g} R_{1 g}, \quad R_{2 g}=-d_{g} S_{2 g}, \quad R_{3 g}=-d_{g} S_{3 g}, \\
T_{2 g}= & -\alpha_{g}\left[R_{g}^{(5)}-4(\pi / 2)^{2} R_{g}^{(3)}-9(\pi / 2)^{4} R_{g}^{(1)}\right], \\
T_{3 g}= & -\alpha_{g}\left[R_{g}^{(6)}-3(\pi / 2)^{2} R_{g}^{(4)}-15(\pi / 2)^{4} R_{g}^{(2)}\right. \\
& \left.-31(\pi / 2)^{6} R_{g}^{(0)}\right], \quad \\
U_{1 g}= & \alpha_{g}\left[R_{g}^{(3)}-3(\pi / 2)^{2} R_{g}^{(1)}\right], \quad T_{1 g}=-d_{g} U_{1 g}, \\
U_{2 g}= & d_{g} T_{2 g}, \quad U_{3 g}=d_{g} T_{3 g} .
\end{aligned}
$$

The corresponding terms for the absorber, $P_{i a}, Q_{i a}, R_{i a}$, $S_{i a}, T_{i a}$, and $U_{i a}$, are obtained by replacing the subscript $g$ with $a$ in $\alpha_{g}, d_{g}$, and $R_{g}^{(n)}$ in the above equations.

\section{ACKNOWLEDGMENT}

We thank Shiro Kaga, Vacuum Optics Corporation of Japan, for helpful discussions of the manufacturing process for multilayer coatings.

\section{REFERENCES}

1. J. J. Fontaine, W. Dietel, and J. C. Diels, "Chirp in a modelocked ring dye laser," IEEE J. Quantum Electron. QE-19, 1467 (1983).

2. J. A. Valdmanis and R. L. Fork, "Design considerations for a femtosecond pulse laser balancing self phase modulation, group velocity dispersion, saturable absorption and saturable gain," IEEE J. Quantum Electron. QE-22, 112 (1986).

3. J. Heppner and J. Kuhl, "Intracavity chirp compensation in a colliding pulse mode-locked laser using thin-film interferometers," Appl. Phys. Lett. 47, 453 (1985).

4. M. Yamashita, M. Ishikawa, K. Torizuka, and T. Sato, "Femtosecond-pulse laser chirp compensated by cavitymirror dispersion," Opt. Lett. 11, 504 (1986).

5. M. Yamashita, K. Torizuka, and T. Sato, "A chirp-compensation technique using incident-angle changes of cavity mirrors in a femtosecond pulse laser," IEEE J. Quantum Electron. QE-23, 2005 (1987).

6. M. Yamashita, S. Kaga, and K. Torizuka, "Chirp-compensation cavity-mirrors with minimal third-order dispersion for use in a femtosecond pulse laser," Opt. Commun. 76, 363 (1990).

7. J. A. Valdmanis, R. L. Fork, and J. P. Gordon, "Generation of optical pulses as short as 27 femtoseconds directly from a laser balancing self-phase modulation, group-velocity disper- 
sion, saturable absorption, and saturable gain," Opt. Lett. 10, 131 (1985).

8. R. S. Miranda, G. R. Jacobovitz, C. H. Brito Cruz, and M. A. F. Scarparo, "Positive and negative chirping of laser pulses shorter than $100 \mathrm{fsec}$ in a saturable absorber," Opt. Lett. 11, 224 (1986)

9. G. H. C. New, "Pulse evolution in mode-locked quasicontinuous lasers," IEEE J. Quantum. Electron. QE-10, 115 (1974).

10. H. A. Haus, "Parameter ranges for cw passive mode locking," IEEE J. Quantum Electron. QE-12, 169 (1976).

11. D. Khühlke, W. Rudolph, and B. Wilhelmi, "Influence of transient absorber gratings on the pulse parameters of passively mode-locked cw dye ring lasers," Appl. Phys. Lett. 42, 325 (1983).

12. J. Herrmann and B. Wilhelmi, Lasers for Ultrashort Light Pulses (North-Holland, Amsterdam, 1987), Chap. 6.

13. S. Silvestri, P. Laporta, and O. Svelto, "The role of cavity dispersion in cw mode-locked lasers," IEEE J. Quantum Electron. QE-20, 533 (1984).

14. O. E. Martinez, R. L. Fork, and J. P. Gordon, "Theory of passively mode-locked lasers for the case of a nonlinear complexpropagation coefficient," J. Opt. Soc. Am. B 2, 753 (1985).

15. H. A. Haus and Y. Silberberg, "Laser mode locking with addition of a nonlinear index," IEEE J. Quantum Electron. QE-22, 325 (1986)

16. F. Salin, P. Grangier, G. Roger, and A. Brun, "Observation of high-order solitons directly produced by a femtosecond ring laser," Phys. Rev. Lett. 36, 1132 (1986).

17. F. Salin, P. Grangier, G. Roger, and A. Brun, "Experimental observation of nonsymmetrical $N=2$ solitons in a femtosecond laser," Phys. Rev. Lett. 60, 569 (1988).
18. F. W. Wise, I. A. Walmsley, and C. L. Tang, "Simultaneous formation of solitons and dispersive waves in a femtosecond ring dye laser," Opt. Lett. 13, 129 (1988).

19. C. Wang, Y. Ishida, and Y. Yamamoto, "Self-phasemodulation-controlled passively mode-locked dye laser," Opt. Lett. 15, 965 (1990).

20. H. Avramopoulos and G. H. C. New, "A numerical model for the study of phase effects in passive mode-locking," Opt. Commun. 71, 370 (1989).

21. H. Avramopoulos and R. L. Fork, "Bandwidth limitation and distinct operating regimes of passively mode-locked dye lasers using strong phase shaping," J. Opt. Soc. Am. B 8, 117 (1990).

22. D. Khühlke, T. Bonkhofer, and D. Von der Linde, "Pulse fluctuations and chirp compensation in colliding-pulse modelocked dye lasers," Opt. Commun. 59, 208 (1986).

23. R. L. Fork, C. H. Brito Cruz, P. C. Becker, and C. V. Shank, "Compression of optical pulses to six femtoseconds by using cubic phase compensation," Opt. Lett. 12, 483 (1987).

24. M. R. X. de Barros, R. S. Miranda, and C. H. Brito Cruz, "Third-order group-velocity dispersion in a colliding-pulse mode-locked dye laser," Opt. Lett. 15, 127 (1990).

25. H. Goto, K. Ueda, S. Hashiguti, and Y. Kawano, "Generation of the range of 20 to $30 \mathrm{fs}$ light pulses by 3-rd order phase dispersion compensation," in Extended Abstracts No. 3 The 37-th Spring Meeting (Japan Society of Applied Physics, Tokyo, 1990), paper 30p-G-10, p. 866.

26. F. Salin, P. Grangier, P. Georges, and A. Brun, "Pulse propagation near zero group-velocity dispersion in a femtosecond dye laser," Opt. Lett. 15, 1374 (1990). 